\title{
Cross-frequency coupling of brain oscillations in studying motivation and emotion
}

\author{
Dennis J. L. G. Schutter • Gennady G. Knyazev
}

Published online: 31 July 2011

(c) The Author(s) 2011. This article is published with open access at Springerlink.com

\begin{abstract}
Research has shown that brain functions are realized by simultaneous oscillations in various frequency bands. In addition to examining oscillations in pre-specified bands, interactions and relations between the different frequency bandwidths is another important aspect that needs to be considered in unraveling the workings of the human brain and its functions. In this review we provide evidence that studying interdependencies between brain oscillations may be a valuable approach to study the electrophysiological processes associated with motivation and emotional states. Studies will be presented showing that amplitude-amplitude coupling between delta-alpha and delta-beta oscillations varies as a function of state anxiety and approach-avoidance-related motivation, and that changes in the association between delta-beta oscillations can be observed following successful psychotherapy. Together these studies suggest that cross-frequency coupling of brain oscillations may contribute to expanding our understanding of the neural processes underlying motivation and emotion.
\end{abstract}

Keywords Alpha waves - Anxiety - Bandwidth ·

Beta waves · Cortex · Cortisol · Cross-frequency coupling · Delta waves - Electroencephalogram · Emotion ·

Limbic system · Motivation · Oscillations ·

Psychopathology $\cdot$ Testosterone $\cdot$ Theta waves

D. J. L. G. Schutter $(\bowtie)$

Experimental Psychology, Helmholtz Institute, Utrecht University, Heidelberglaan 2, 3584 CS Utrecht, The Netherlands e-mail: d.schutter@uu.nl

G. G. Knyazev

Institute of Physiology, Siberian Branch of the Russian Academy of Medical Sciences, Timakova str. 4, Novosibirsk, Russia e-mail: knyazev@physiol.ru

\section{Introduction}

Ever since the successful recording of $10 \mathrm{~Hz}$ brain oscillations over the intact scalp of human subjects by Berger in 1929, the study of brain oscillations in the electroencephalogram (EEG) has become an increasingly important focus of interest in studying brain-behavior relationships. Brain oscillations are the result of the continue waxing and waning of electric field potentials generated simultaneously in a large number of vertically oriented apical dendrites of pyramidal nerve cells in the cerebral cortex (Niedermeyer and Lopes da Silva 1999). In spite of having a cortical read-out brain oscillations come about by direct or indirect interactions with other local and distal structures subsequently forming complex networks.

\section{Brain oscillations}

Evolutionary views on human brain development and complexity suggest that the brain is comprised of several phylogenetically distinct networks (MacLean 1990). Notably, each network has its own natural frequency by which the network operates. Furthermore, it has been proposed that more complex networks operate using higher frequency ranges. In line, slow wave activity corresponding to delta $(1-3 \mathrm{~Hz})$ and theta $(4-7 \mathrm{~Hz})$ frequency range stems from the 'evolutionary older' subcortical structures (including the brainstem and septo-hippocampal complex). In agreement with this view, electric stimulation of the brain-stem ascending reticular activating system (ARAS) elicits 1-4 Hz (delta) cortical responses (Guyton 1976; but see Knyazev 2011), while electric stimulation of limbic areas evoke distinct $7 \mathrm{~Hz}$ (theta) activity (Gray 1982). Fast wave activity in the alpha $(8-12 \mathrm{~Hz})$ and beta $(13-30 \mathrm{~Hz})$ 
frequency range, on the other hand, is suggested to find its origin in thalamo-cortical and cortico-cortical circuits respectively (Knyazev and Slobodskaya 2003; Knyazev et al. 2004; Robinson 1982, 1999, 2000, 2001). In line with this view, Michel et al. (1992) used 3-dimensional dipole modeling to show that the source of delta oscillations was deepest and most anterior, theta oscillations more posterior and less deep, alpha oscillations most posterior and highest on the vertical dimension. Thus, dominant rhythms shift from low frequency to higher frequency with increasing complexity of the brain, yet lower frequencies are suggested to be preserved and arguably reflect neural systems that are evolutionary more ancient. Although mostly speculative of nature, this conceptual framework may be helpful in the interpretation of findings on cross-frequency coupling and to guide further research.

The functional relevance of brain oscillations and their accompanying frequency underwent a thorough change in course of time from admitting that they are useful only for making inferences about global states of sleep and wakefulness (e.g., Duffy 1962; Thayer 1989) to acknowledging that oscillations may play a special role in large scale synchronization of brain functions (Buzsaki and Draguhn 2004; Salinas and Sejnowski 2001; Singer 1999). Considerable evidence links different frequency brain oscillations with a range of cognitive processes, emotional states, and behaviors (Basar 1999; Cantero and Atienza 2005; Klimesch 1999; Knyazev 2007; Nunez 2000; Varela et al. 2001).

\section{Indices of cross-frequency coupling}

Importantly, studying these brain-behavior relationships is usually limited to examining oscillations in a pre-specified band, whereas brain functions are believed to be realized by simultaneous oscillations in various frequency bands (Basar 2006). Additionally, studying interactions and relations between the different frequency bandwidths provides a logical next step in unraveling the workings of the human brain and its functions.

Indeed, more recent studies report on statistical relations among activities in different frequency bands. These studies may be roughly divided into several categories. Firstly, a range of methods have been suggested to measure phase coordination between different frequencies. To explore phase coupling between different frequencies, higher order statistics are commonly used. The calculation of bicoherence is an extension of second order statistics used in determining signal properties in the time and frequency domains, in the form of Fourier transform (Schack et al. 2002). This measure mixes phase and amplitude relations between signals or the phase-amplitude relationship between different frequencies. In order to study a pure phase-phase relationship, that is phase synchronizations, across frequencies, a method to estimate time varying quadratic phase coupling of electrophysiological signals, the biphase locking value was introduced (Darvas et al. 2009). Another method is termed " $\mathrm{n}: \mathrm{m}$ " phase synchronization, and involves the synchrony between phase values at one frequency band and phase values at an integer multiple of another frequency band (Tass et al. 1998; Palva et al. 2005; Schack and Weiss 2005). This method is useful for examining amplitude-independent phase-locking, but is limited to coupling among integer-multiple frequency bands (e.g., $10,20,40 \mathrm{~Hz}$ ). In general, cross-frequency phase coordination in the human brain suggests nonlinear interactions, and it is speculated that such interactions play a crucial role in the coordination of complex cortical computations (Darvas et al. 2009). Another type of cross-frequency coupling is called phase-amplitude coupling and refers to the synchronization of oscillation power fluctuations with the phase of a slower oscillation (Jensen and Colgin 2007). For example, in the entorhinal and prefrontal cortices, the amplitude of gamma oscillations increases during specific phases of theta (Chrobak and Buzsaki 1998; Canolty et al. 2006; Demiralp et al. 2007), amplitude modulation of occipital high-frequency oscillations in the gamma range may be phase locked to a slow-frequency oscillation in the delta frequency range (Handel and Haarmeier 2009). Both phase-phase and phase-amplitude cross-frequency coupling have been shown to relate to specific cortical areas and specific stages of information processing and have received much attention in recent works. Although another type of cross-frequency relationship called amplitude-amplitude coupling has received less attention, this review will provide evidence that the amplitude-amplitude coupling may reflect some global properties of the brain oscillatory systems, which might be relevant to the description of stable individual differences, or emotional and motivational states.

Amplitude-amplitude associations between different frequency EEG bands could be studied both in the within(i.e., across a number of states within each individual) and between- (i.e., across a sample of subjects) subject domain. Given the large number of EEG variables (i.e., a multitude of cortical sites and frequency bands), these associations have been frequently analyzed by means of multivariate techniques, such as factor analysis, or principal component analysis. These techniques are used as a means for reducing the amount of correlated data, with no a priori predictions as to the relationship between different frequency bands. As a result, cross-frequency associations were frequently mixed with spatial associations. For instance, a study with 85 healthy adolescents by Volkow et al. (1987) revealed three factors that accounted for most of the variability in the data: (1) low versus high frequency, (2) beta frequency, and (3) frontal versus posterior factor. 


\section{Interrelations with motivation, emotion and personality}

In his pioneering work Robinson (1982, 1999, 2000, 2001) used factor analysis to test predictions of his arousability theory. The theory posits that the thalamo-cortical system exerts a generally inhibitory effect on the brainstem system and, hence, amplitudes of alpha and delta waves should be inversely related to each other (Robinson 1999). The strength of this negative relationship reflects general cortical arousability and is associated with personality characteristics (Robinson 2001). Although some assumptions of this theory are speculative and do not seem to be supported by more recent findings, Robinson has been able to confirm its main predictions empirically. He performed factor analysis of filtered in alpha, theta, and delta bands auditory evoked potentials registered in a relatively large sample of aged subjects. Considering the unrotated factor solution, Robinson showed that overwhelming part of the variance could be explained by the first component showing high positive loadings from all three bands. The second component provided an opportunity to observe negative relations between variables. Here, as predicted, negative covariance between delta and alpha bands emerged. The behavioral relevance of this inverse relationship between alpha and delta waves to personality was confirmed by the discovery of its lower magnitude in individuals with high scores on extraversion and neuroticism (Robinson 2001).

Knyazev et al. $(2003,2004)$ repeated this analysis for recorded in resting condition spontaneous EEG and obtained similar results. It appeared that, as compared to adults, children had significantly lower scores on the factor measuring negative relationship between alpha and delta powers. These factor scores were positively related to psychometric measures of neuroticism, behavioral inhibition, and emotional symptoms and were inversely related to measures of extraversion, behavioral activation, and conduct problems. This measure showed trait-like properties being uniformly higher in high trait anxiety subjects independently of experimental manipulations (Knyazev et al. 2006). A within-subject measure of delta-alpha anticorrelation was explored by Knyazev and Slobodskaya (2003) and Knyazev et al. (2004). This measure was positively related to relative alpha power, inversely to relative delta and theta power. In addition, the within-subject measure showed a moderate positive relation to the respective between-subject measure and with psychometric measures of behavioral inhibition. Such a pattern of correlations implies that the within- and the between-subject estimates measure a comparable construct that may underlie a mechanism involving the increase of alpha oscillations and the inhibition of delta and theta oscillations (Knyazev et al. 2004). Noteworthy, most of these associations were not significant in children and could be ascribed to immaturity of this hypothetical mechanism. Apart from these factoranalytic studies, a large body of evidence coming from different experimental manipulations and observations in psychopathological and juvenile populations confirms a general tendency for alpha and slower (delta and theta) frequencies to be inversely related to each other, with a prevalence of slow frequencies in some pathological conditions and early developmental stages being associated with impulsivity and disinhibition (for a review see Knyazev 2007). For example, delta oscillations have been associated with the approach-related behavior and reward sensitivity (Knyazev et al. 2003; Schutter and van Honk 2004). Interestingly, the steroid hormone testosterone has been associated with approach-related motivational tendencies, reward sensitivity and aggression (van Honk et al. 2004). Testosterone is the end-product of the hypothalamic-pituitarygonadal (HPG-) axis and its target regions include widely distributed steroid networks in the limbic system (Wood 1996) that modulate core motivational and emotional drives. In a previous placebo-controlled study van Honk et al. (2004) showed that a single administration of testosterone reduced punishment sensitivity and increased reward dependency as subjects made more disadvantageous, risky decisions in the Iowa gambling task. In an additional resting state EEG study significant increases in frontal delta oscillations were observed after a single administration of testosterone (Schutter and van Honk 2004). All these data fit the proposal that delta oscillations might be somehow related to the activity of motivational brain circuits (Knyazev 2007). In addition to the significant increase in delta oscillations after testosterone administration, Schutter and van Honk (2004) also found a reduction of coupling between frontal delta and beta oscillations. This finding initiated a series of studies investigating the delta-beta coupling in different groups of subjects and in different conditions. An association study yielded comparable results as males who were high in baseline salivary testosterone exhibited significantly reduced frontal delta-beta coupling compared to those low in testosterone (Miskovic and Schmidt 2009). More recently, a functional magnetic resonance imaging (fMRI) study showed that exogenous elevations of testosterone levels comparable to the previous studies reduce functional cross-talk between the amygdala and orbitofrontal cortex during passive face viewing (van Wingen et al. 2010). In contrast, the stress-related hormone cortisol is a steroid endocrine marker of behavioral inhibition and anxiety (Kalin et al. 1998). Schutter and van Honk (2005) demonstrated that higher levels of cortisol were associated with increased frontal delta-beta coupling in healthy subjects. Furthermore, a single administration of cortisol in healthy volunteers was found to significantly increase the coupling between delta and beta oscillations (van Peer et al. 2008), providing support for the modulatory effects of 
steroid hormones on cross-frequency coupling, as well as the proposed mutually inhibitory function between cortisol and testosterone (Viau 2002).

This series of steroid hormone-EEG studies provide evidence that the magnitude of the correlation between powers of slow and fast waves can be manipulated by factors that are associated with the subject's physiological and subsequent mental state. Indeed, a statistical significant relationship, $\operatorname{rho}(51)=-0.33: p=0.02$, has been observed between reduced individual delta-beta coupling based on the cross-frequency correlation computed from a 4 min baseline recording and increased scores on the BussPerry physical aggression scale (Schutter 2011). Moreover, Knyazev et al. (2005) showed that relative to baseline the delta-beta coupling increased when subjects were expecting bad news but did not change when they expected good news. This increase was more pronounced in participants with higher levels of trait anxiety. Miskovic et al. (2010b) showed that a high socially anxious group from normative population showed significantly greater delta-beta coupling than a low socially anxious group while anticipating public speaking. A number of studies analyzed the delta-beta coupling in groups with anxiety-related pathological conditions. A study by Miskovic et al. (2009) provides evidence that individuals diagnosed with social anxiety disorder (generalized subtype) exhibit high levels of EEG delta-beta coupling during the anticipation of a self-presentation task, whereas cognitive behavioural therapy significantly reduces the anticipatory delta-beta coupling. Moreover, higher frontal delta-beta coupling was registered in children of parents with social phobia as compared to children of healthy parents (Miskovic et al. 2010a). In addition to replicating their earlier findings Miskovic et al. (2011) showed that successful psychotherapy in social phobic patients was associated with decreases in delta-beta coupling paralleled by reductions in anxiety symptoms at rest and during speech anticipation. In this study twentyfive subjects diagnosed with social anxiety disorder underwent 12 weekly sessions of standardized group cognitive behavioral therapy. While increased delta-beta coupling was observed in the clinical group during the pretreatment phase as compared to control participants with low social anxiety, reductions in symptom severity as well as normalization of the delta-beta coupling were observed during the treatment and post-treatment phase of the study. Finally, Velikova et al. (2010), on the other hand, have found lower delta-beta coupling in patients with obsessive-compulsive disorder than in age- and sex-matched controls. According to the authors, obsessive-compulsive disorder is related not only to higher activity levels in frontal cortical networks, but also to a fronto-subcortical functional disconnection. In Table 1 a summary of the studies and their main findings can be found.
In sum, evidence from several lines of fundamental and clinical neuroscientific research demonstrates that crossfrequency EEG relations are associated with various aspects of human behavior, providing a possible indirect electrophysiological correlate for cortico-subcortical interactions (Knyazev 2007; Robinson 1982; Schutter et al. 2006).

\section{Discussion and future directions}

Empirical studies have demonstrated that delta-alpha and delta-beta frequency coupling in the human EEG are associated with phenomenology, behavior, and hormones. Despite the available evidence, the cross-frequency coupling approach in its present form has several conceptual and methodological issues that will be discussed in more detail below.

The frequency information buried within the EEG signal reveals an important physiological property by which the brain operates as rhythmic oscillations constitute an important organizing principle of neural functions. Extracting the different frequency components from the signal provides a means to establish an association between brain states and function. Traditionally, high frequency low amplitude (e.g., beta) oscillations are associated with a highly active cerebral cortex (e.g., awake and alert), whereas slow frequency high amplitude (e.g., delta) oscillations are linked to low aroused and relative inactive cortical state (e.g., deep sleep). Additionally, alpha oscillations have been interpreted as being inversely related to cortical activity. One line of evidence comes from the observation of alpha waves in the EEG signal over the occipital cortex when subjects have their eyes closed and no visual information is being processed by the visual system (Berger 1929). There is evidence to suggest that the alpha oscillations find its origins in thalamo-cortical loops. Beta oscillations and even higher frequencies are proposed to have a cortico-cortical basis indexing local information processing and have been associated with active inhibition processes that are possibly mediated by GABA-ergic interneurons (Jensen et al. 2005). Theta-oscillations have been associated with the subcortical septo-hippocampal region and the anterior cingulate cortex (ACC) and are connected to memory, anxiety and punishment learning (Gray 1982). Finally, delta oscillations have been linked to the locus coeruleus and the brain stem ascending reticular activating system (Leung and Yim 1993; Robinson 1999). On the behavioral level delta waves have been associated with approach-related action and reward (Knyazev 2007). Neuroimaging studies in humans have demonstrated an anatomical correspondence between the putative location of delta wave generation and the projections of the mesencephalic dopamine system to the cerebral cortex. Indeed, studies using dipole modeling place the site of cortical delta 
Table 1 Overview studies

\begin{tabular}{|c|c|c|c|}
\hline Study & Population & $\begin{array}{l}\text { Cross-frequency } \\
\text { coupling }\end{array}$ & Main finding \\
\hline Robinson (2001) & Healthy & Delta-alpha & $\begin{array}{l}\text { Lower coupling in individuals scoring high on extraversion } \\
\text { and neuroticism }\end{array}$ \\
\hline Knyazev and Slobodskaya (2003) & Healthy & Delta-alpha & $\begin{array}{l}\text { Higher coupling associated with increased behavioral } \\
\text { inhibition }\end{array}$ \\
\hline Knyazev et al. (2003) & Healthy & Delta-alpha & $\begin{array}{l}\text { Lower coupling associated with increased Extraversion } \\
\text { and behavioural activation (BAS) }\end{array}$ \\
\hline Schutter and van Honk (2004) & Healthy & Delta-beta & Reduced coupling after testosterone administration \\
\hline Schutter and van Honk (2005) & Healthy & Delta-beta & Higher basal cortisol levels associated with increased coupling \\
\hline Knyazev et al. (2005) & Healthy & Delta-beta & Increased coupling under situations of uncertainty \\
\hline Knyazev et al. (2006) & Healthy & Delta-beta & Increased coupling during state anxiety \\
\hline Knyazev and Slobodskoj-Plusnin (2007) & Healthy & Theta-gamma & $\begin{array}{l}\text { Increased coupling under situation of reward in high BAS } \\
\text { subjects }\end{array}$ \\
\hline van Peer et al. (2008) & Healthy & Delta-beta & Increased coupling after cortisol administration \\
\hline Miskovic and Schmidt (2009) & Healthy & Delta-beta & $\begin{array}{l}\text { Higher basal testosterone levels associated with reduced } \\
\text { coupling }\end{array}$ \\
\hline Miskovic et al. $(2009,2011)$ & Patients & Delta-beta & $\begin{array}{l}\text { Reduced coupling following successful behavioral therapy } \\
\text { of social anxiety }\end{array}$ \\
\hline Miskovic et al. (2010a) & Patients & Delta-beta & Increased coupling in children of parents with social phobia \\
\hline Miskovic et al. (2010b) & Patients & Delta-beta & $\begin{array}{l}\text { Increased coupling during speech anticipation in patients with } \\
\text { social anxiety disorder }\end{array}$ \\
\hline Velikova et al. (2010) & Patients & Delta-beta & $\begin{array}{l}\text { Reduced coupling in patients with obsessive compulsive } \\
\text { disorder }\end{array}$ \\
\hline Knyazev (2011) & Healthy & Delta-beta & Increased coupling during state anxiety \\
\hline Putman (2011) & Healthy & Delta-beta & Higher coupling associated with attentional bias towards threat \\
\hline Schutter (2011) & Healthy & Delta-beta & $\begin{array}{l}\text { Lower coupling associated with higher physical aggression } \\
\text { scores }\end{array}$ \\
\hline
\end{tabular}

wave generation in anterior medial frontal cortex (Michel et al. 1992; Michel et al. 1993). Studies correlating positron emission tomography (PET) and EEG also indicate a positive correlation between waking delta and PET metabolism in the medial frontal cortex (Alper et al. 1995, 1998, 2006). More recently, Wacker et al. (2009) have shown that delta activity in the rostral anterior cingulate cortex inversely correlates with the nucleus accumbens responses to reward and is associated with higher anhedonia scores. Importantly, all these data show that delta activity increases in states associated with a need for some reinforcement and decreases when this reinforcement is obtained. It should furthermore be noted that these frequencies are all present in the awake EEG of human subjects. Moreover, delta and theta oscillations originate in the subcortical motivational circuits, while the generation of alpha and beta oscillations are suggested to be more closely related to cortical structures that are responsible for higher order mental functions including cognitive regulation. However, the precise functional meaning of the different frequency bands remains to be determined. It should be emphasized that although we review works studying the delta-alpha and the delta-beta interactions together under the concept of cross-frequency coupling, they probably reflect quite different phenomena. Moreover, the measures that were used in these two kinds of studies are also different. Part of the answer to the previous question may come from studies that will establish the neural generators of these oscillations and how the coupling between the different frequency bands comes about. In most of earlier studies, spectral power measures were averaged across electrode locations and inferences about the localization of the effect could not be made. Some studies, however, suggest that this effect might relate to the prefrontal cortex (Schutter et al. 2006; Miskovic and Schmidt 2009; van Wingen et al. 2010). Velikova et al. (2010) used source localization technique to reveal possible subcortical localization of the observed effects. Whereas beta oscillations were primarily found in the cerebral cortex, delta oscillations appear to originate from the insular cortex, a structure that is directly connected to the subcortical structures including the brain stem. However, without the proper methods to determine the neural sources responsible it is impossible to draw any definite conclusion concerning the origin of these brain rhythms. More studies with a more meticulous analysis of precise localization of the cross-frequency coupling are needed. Because, due to volume conduction, the EEG signal does not 
give precise localization of observed effects, more sophisticated analysis methods, such as independent component analysis and interleaving functional neuroimaging with EEG recordings, may prove useful in this effort.

The majority of studies show that the delta-beta coupling is increased during anxiogenic situations, after administration of potentially anxiogenic drugs, and in groups with presumably higher anxiety levels (but see Velikova et al. 2010). The question arises whether this effect is limited to anxiety or can be observed in other motivationally relevant states as well. In other words, what is the specificity of the cross-frequency coupling? To our knowledge, there are only two studies currently available that suggest that the cross-frequency coupling extends to other psychological states as well. In the first study evidence was found that in reward sensitive subjects the thetagamma $(30-40 \mathrm{~Hz})$ coupling increases in situations that presumably elicit positive emotional arousal (Knyazev and Slobodskoj-Plusnin 2007). The second study refers to the finding that physical aggression is linked to reductions in delta-beta coupling (Schutter 2011).

A possible reason that relations with alpha oscillations are not always observed may be due to the fact that the cross-frequency relations are sometimes studied during relative active states and under these conditions alpha wave activity as a rule is desynchronized. Moreover, in resting state conditions where alpha waves are present in the signal, activity recorded from the anterior scalp sites from which the cross-frequency coupling is often recorded could be potentially confounded by activity that originates from the posterior sites due to volume conduction of the scalp. As a result interrelations involving alpha oscillations over the frontal regions could thus be masked by alpha oscillations which have a different origin. It should also be noted that using extracranial EEG recordings the frequency range of activity that can be measured from the surface is limited and therefore not suitable to study brain signals in very fast frequencies (e.g., $>100 \mathrm{~Hz}$ ).

Additionally, the cross-frequency coupling does not need to behave in a linear fashion per se. There is ample evidence from EEG research showing the importance of studying non-linear relations between electric brain signals in understanding function and behaviour (Darvas et al. 2009). For example, Darvas et al. (2009) showed that interactions between distal brain regions can be understood in terms of quadratic relationships between different frequency bandwidths.

The reported cross-frequency couplings are based on correlational computations and consequently do not provide information on the direction of information flow. It remains therefore unclear whether slow or fast waves drive the coupling and under what circumstances. There is some evidence that delta waves are capable of influencing the delta-beta coupling. For example, the observed increases in delta oscillations paralleled by a reduction in the coupling between delta and beta oscillations after a single administration of testosterone suggest a modulation of the crossfrequency coupling driven by a change in delta wave activity (Schutter and van Honk 2004). In another study evidence was provided that the delta-beta coupling may be more sensitive to variations in delta activity than to beta activity (Schutter et al. 2006). Additionally, Knyazev et al. (2005) found that delta activity measured before induction of an anxious state predicted beta activity after the induction, but not vice versa. Importantly, neither the strength nor the sign of the coupling provides information whether the interaction reflects a bottom-up or top-down process that is either inhibitory or excitatory. It has been proposed that anti-correlations may reflect a top-down inhibitory process, but due to the limitations of the linear approach the anti-correlation may also reflect a bottom-up excitatory process. Incorporating additional phase information or introducing phase lags in computing the correlations as an index of the cross-frequency coupling may be helpful in unraveling directional information of the coupling.

Except for one published study by Knyazev and Slobodskaya (2003) all studies reporting on the cross-frequency coupling in the existing literature are based on a sample based (between-subjects) approach and extrapolated to the individual (within-subject) domain (cf. Ochsner et al. 2002). This extrapolation raises the issue of construct validity as to whether or not the sample-based approach (between-subjects) can be used to index the individual (within-subject) level. Knyazev et al. (2004) found good agreement between the individual (within-subject) and sample-based (between-subjects) measures of delta-alpha anti-correlation. Moreover, in line with the sample-based approach an association between lower individual (withinsubject) delta-beta coupling and increases in physical aggression has recently been observed (Schutter 2011). In spite of these promising findings, future studies are needed to establish the relation between individual (within-subject) cross-frequency coupling and behaviour, as well as research that examine the correspondence between the sample-based (between-subjects) and individual-based (within-subject) approach in more detail.

Finally, the frequency correspondence between the discussed amplitude-amplitude coupling and the well documented phase-amplitude coupling may be more than just a coincidence. In fact, the same mechanism may underlie both effects. Furthermore, phase-phase cross frequency relations may also provide another way of studying the electrophysiological properties underlying brain function and behaviour (Darvas et al. 2009). The simultaneous investigation of these different approaches in the same subjects under comparable conditions may bring forth 
exciting new insights into the operational dynamics of the human brain.

A recent study by Knyazev (2011) attempted to clarify some of these questions. In this study, independent component analysis and source localization techniques were used to identify temporally correlated spatial patterns that most reliably show the phenomenon of between-subject delta-beta correlation. Results of this analysis show that in an anxiogenic situation, the delta-beta correlation increases in a network of cortical areas which includes the orbitofrontal and the ACC as its main nodes. The increase of the correlation is accompanied by an increase of delta power and connectivity in the same cortical regions. Analysis of the within-subject delta-beta amplitude-to-amplitude and phase-to-amplitude coupling showed that in an anxiogenic situation, in subjects with higher scores on state anxiety the couplings also tend to increase in the same set of cortical areas. This pattern of results suggests that the increase of delta power in medial frontal and orbitofrontal cortices during an anxiogenic situation in high state anxiety subjects, might be the driving force behind the (betweensubject) cross-frequency coupling and that this coupling is closely related to the within-subject cross-frequency amplitude-amplitude and phase-amplitude coupling.

\section{Conclusion}

The introduction of the EEG by Berger in 1929 hallmarked an important step forward in studying brain-behavior relationships. Research has shown that brain functions are realized by simultaneous oscillations in various frequency bands (Basar 2006). In addition to examining oscillations in pre-specified bands, interactions and relations between the different frequency bandwidths is another important aspect that needs to be considered in unraveling the workings of the human brain and its functions. The aim of the present overview was to show that the concept of crossfrequency relations may be a useful and valuable approach to study the neural processes associated with motivation and emotion. The first series of studies have provided evidence that the cross-frequency coupling between slow and fast wave oscillations is sensitive to steroid hormones and associated with varying motivational states in which normal and pathological forms of anxiety have received most attention. Recent data showing that changes in the cross-frequency coupling can be observed following successful psychotherapy is notable (Miskovic et al. 2011). Further research designed to extend these findings to other (pathological) conditions and to explore the specificity and sensitivity of the cross-frequency coupling in the healthy and clinical population is necessary. In spite of the remaining issues surrounding the cross-frequency approach, studying interrelations between different brain wave oscillations may yield important new insights into the organization and dynamics of human brain functions.

Acknowledgments Dennis J. L. G. Schutter was supported by an Innovational Research Grant (VIDI 451-07-012) from the Netherlands Organization for Scientific Research (NWO). Gennady G. Knyazev was supported by a grant from the Russian Foundation for Basic Research (RFBR) No. 11-06-00041-a.

\section{Conflict of interest None.}

Open Access This article is distributed under the terms of the Creative Commons Attribution Noncommercial License which permits any noncommercial use, distribution, and reproduction in any medium, provided the original author(s) and source are credited.

\section{References}

Alper, K. R., Gunther, W., Prichep, L. S., John, E. R., \& Brodie, J. (1998). Correlation of qEEG with PET in schizophrenia. Neuropsychobiology, 38, 50-56.

Alper, K. R., John, E. R., Brodie, J., Günther, W., Daruwala, R., \& Prichep, L. S. (2006). Correlation of PET and qEEG in normal subjects. Psychiatry Research, 146, 271-282.

Alper, K. R., Prichep, L. S., John, E. R., Kowalik, S., \& Merkin, H. (1995). QEEG in cocaine withdrawal: Possible neurophysiological implications regarding the generation of slow EEG activity. 6th International Congress of the International Society for Brain Electromagnetic Topography (ISBET., Tokushima, Japan, Abstract No S-2-G.

Basar, E. (1999). Brain function and oscillations. II. Integrative brain function. Neurophysiology and cognitive processes. Berlin: Springer.

Basar, E. (2006). The theory of the whole-brain-work. International Journal of Psychophysiology, 60, 133-138.

Berger, H. (1929). Über das elecktroenzephalogramm des menschen I. Archive Psychiatrie (Nervenkrankheiten), 87, 527-570.

Buzsaki, G., \& Draguhn, A. (2004). Neuronal oscillations in cortical networks. Science, 304, 1926-1929.

Canolty, R. T., Edwards, E., Dalal, S. S., Soltani, M., Nagarajan, S. S., Kirsch, H. E., et al. (2006). High gamma power is phase-locked to theta oscillations in human neocortex. Science, 313, 1626-1628.

Cantero, J. L., \& Atienza, M. (2005). The role of neural synchronization in the emergence of cognition across the wake-sleep cycle. Reviews in the Neurosciences, 16, 69-83.

Chrobak, J. J., \& Buzsaki, G. (1998). Gamma oscillations in the entorhinal cortex of the freely behaving rat. Journal of Neuroscience, 18, 388-398.

Darvas, F., Miller, K. J., Rao, R. P. N., \& Ojemann, J. G. (2009). Nonlinear phase-phase cross-frequency coupling mediates communication between distant sites in human neocortex. Journal of Neuroscience, 29, 426-435.

Demiralp, T., Bayraktaroglu, Z., Lenz, D., Junge, S., Busch, N. A., Maess, B., et al. (2007). Gamma amplitudes are coupled to theta phase in human EEG during visual perception. International Journal of Psychophysiology, 64, 24-30.

Duffy, E. (1962). Activation and behavior. New York: Wiley.

Gray, A. (1982). The neuropsychology of anxiety: An enquiry into the septo-hippocampal system. Oxford: University Press.

Guyton, A. C. (1976). Organ physiology: Structure and function of the nervous system. London: W. B. Saunders. 
Handel, B., \& Haarmeier, T. (2009). Cross-frequency coupling of brain oscillations indicates the success in visual motion discrimination. Neuroimage, 45, 1040-1046.

Jensen, O., \& Colgin, L. L. (2007). Cross-frequency coupling between neuronal oscillations. Trends in Cognitive Sciences, 11, 267-269.

Jensen, O., Goel, P., Kopell, N., Pohja, M., Hari, R., \& Ermentrout, B. (2005). On the human sensorimotor-cortex beta rhythm: Sources and modeling. Neuroimage, 26, 347-355.

Kalin, N. H., Larson, C., Shelton, S. E., \& Davidson, R. J. (1998). Asymmetric frontal brain activity, cortisol, and behavior associated with fearful temperament in rhesus monkeys. Behavioral Neuroscience, 112, 286-292.

Klimesch, W. (1999). EEG alpha and theta oscillations reflect cognitive and memory performance: A review and analysis. Brain Research Reviews, 29, 169-195.

Knyazev, G. G. (2007). Motivation, emotion, and their inhibitory control mirrored in brain oscillations. Neuroscience and Biobehavioral Reviews, 31, 377-395.

Knyazev, G. G. (2011). Cross-frequency coupling of brain oscillations: An impact of state anxiety. International Journal of Psychophysiology, 80, 236-245.

Knyazev, G. G., Savostyanov, A. N., \& Levin, E. A. (2005). Uncertainty, anxiety and brain oscillations. Neuroscience Letters, 387, 121-125.

Knyazev, G. G., Schutter, D. J. L. G., \& van Honk, J. (2006). Anxious apprehension increases coupling of delta and beta oscillations. International Journal Psychophysiology, 61, 283-287.

Knyazev, G. G., \& Slobodskaya, H. R. (2003). Personality trait of behavioral inhibition is associated with oscillatory systems reciprocal relationships. International Journal Psychophysiology, 48, 247-261.

Knyazev, G. G., Slobodskaya, H. R., Safronova, M. V., Sorokin, O. V., Goodman, R., \& Wilson, G. D. (2003). Personality, psychopathology and brain oscillations. Personality and Individual Differences, 35, 1331-1349.

Knyazev, G. G., Slobodskaya, H. R., \& Wilson, G. D. (2004). Personality and brain oscillations: Developmental aspects. In S. P. Shohov (Ed.), Advances in psychology research (Vol. 29, pp. 3-34). New York: Nova Science Publishers.

Knyazev, G. G., \& Slobodskoj-Plusnin, J. Y. (2007). Behavioural approach system as a moderator of emotional arousal elicited by reward and punishment cues. Personality and Individual Differences, 42, 49-59.

Leung, L. S., \& Yim, C. Y. (1993). Rhythmic delta-frequency activities in the nucleus accumbens of anesthetized and freely moving rats. Canadian Journal of Physiology and Pharmacology, 71, 311-320.

MacLean, P. D. (1990). The triune brain and evolution. New York: Plenum Press.

Michel, C. M., Henggeler, B., Brandeis, D., \& Lehmann, D. (1993). Localization of sources of brain alpha/theta/delta activity and the influence of the mode of spontaneous mentation. Physiological Measures, 14, 21-26.

Michel, C. M., Lehmann, D., Henggeler, B., \& Brandeis, D. (1992). Localization of the sources EEG delta, theta, alpha and beta frequency bands using the FFT dipole approximation. Electroencephalography and Clinical Neurophysiology, 82, 38-44.

Miskovic, V., Ashbaugh, A. R., Santesso, D. L., McCabe, R. E., Antony, M. M., \& Schmidt, L. A. (2010a). Frontal brain oscillations and social anxiety: A cross-frequency spectral analysis during baseline and speech anticipation. Biological Psychology, 83, 125-132.

Miskovic, V., Campbell, M. J., Santesso, D. L., van Ameringen, M., Mancini, C., \& Schmidt, L. A. (2010a). Frontal brain oscillatory coupling in children of parents with social phobia: A pilot study.
Journal of Neuropsychiatry and Clinical Neurosciences, 23, 111-114.

Miskovic, V., Moscovitch, D. A., McCabe, R. E., Antony, M. M., \& Schmidt, L. A. (2011). Changes in EEG cross-frequency coupling during cognitive behavioral therapy for social anxiety disorder. Psychological Science, 22, 507-516.

Miskovic, V., Moscovitch, D. A., Senn, J., McCabe, R. E., Antony, M. M., \& Schmidt, L. A. (2009). Neural correlates of cognitive behavioral therapy in social anxiety disorder: Evidence from frontal brain oscillatory coupling [Abstract]. Society for Neuroscience Abstracts.

Miskovic, V., \& Schmidt, L. A. (2009). Frontal brain oscillatory coupling among men who vary in salivary testosterone levels. Neuroscience Letters, 464, 239-242.

Niedermeyer, E., \& Lopes da Silva, F. (1999). Electroencephalography: Basic principles, clinical applications and related fields (fourth edition ed.). Baltimore: Lippincott Williams \& Wilkins.

Nunez, P. L. (2000). Toward a quantitative description of large-scale neocortical dynamic function and EEG. Behavioral and Brain Sciences, 23, 371-398.

Ochsner, K. N., Bunge, S. A., Gross, J. J., \& Gabrieli, J. D. (2002). Rethinking feelings: An fMRI study of the cognitive regulation of emotion. Journal of Cognitive Neuroscience, 14, 1215-1229.

Palva, J. M., Palva, S., \& Kaila, K. (2005). Phase synchrony among neuronal oscillations in the human cortex. Journal of Neuroscience, 25, 3962-3972.

Putman, P. (2011). Resting state EEG delta-beta coherence in relation to anxiety, behavioral inhibition, and selective attentional processing of threatening stimuli. International Journal of Psychophysiology, 80, 63-68.

Robinson, D. L. (1982). Properties of the diffuse thalamocortical system and human personality: A direct test of Pavlovian/ Eysenckian theory. Personality and Individual Differences, 3, $1-16$.

Robinson, D. L. (1999). The technical, neurological and psychological significance of 'alpha', 'delta' and 'theta' waves confounded in EEG evoked potentials: A study of peak latencies. Clinical Neurophysiology, 110, 1427-1434.

Robinson, D. L. (2000). The technical, neurological and psychological significance of 'alpha', 'delta' and 'theta' waves confounded in EEG evoked potentials: A study of peak amplitudes. Personality and Individual Differences, 28, 673-693.

Robinson, D. L. (2001). How brain arousal systems determine different temperament types and the major dimensions of personality. Personality and Individual Differences, 31, 1233-1259.

Salinas, E., \& Sejnowski, T. J. (2001). Correlated neuronal activity and the flow of neural information. Nature Review Neuroscience, 2, 539-550.

Schack, B., Vath, N., Petsche, H., Geissler, H. G., \& Moller, E. (2002). Phase-coupling of theta-gamma EEG rhythms during short-term memory processing. International Journal of Psychophysiology, 44, 143-163.

Schack, B., \& Weiss, S. (2005). Quantification of phase synchronization phenomena and their importance for verbal memory processes. Biological Cybernetics, 92, 275-287.

Schutter, D. J. L. G. (2011). Reduced within subject delta-beta crossfrequency coupling is associated with higher physical aggression. Unpublished data.

Schutter, D. J. L. G., Leitner, C., Kenenmans, J. L., \& van Honk, J. (2006). Electrophysiological correlates of cortico-subcortical interaction: A cross-frequency spectral EEG analysis. Clinical Neurophysiology, 117, 381-387.

Schutter, D. J. L. G., \& van Honk, J. (2004). Decoupling of midfrontal delta-beta oscillations after testosterone administration. International Journal of Psychophysiology, 53, 71-73. 
Schutter, D. J. L. G., \& van Honk, J. (2005). Salivary cortisol levels and the coupling of midfrontal delta-beta oscillations. International Journal of Psychophysiology, 55, 127-129.

Singer, W. (1999). Neuronal synchrony: A versatile code for the definition of relations? Neuron, 24, 49-65.

Tass, P., Rosenblum, M. G., Weule, J., Kurths, J., Pikovsky, A., Volkmann, J., et al. (1998). Detection of n:m phase locking from noisy data: Application to magnetoencephalography. Physics Review Letters, 81, 3291-3294.

Thayer, R. E. (1989). The biopsychology of mood and arousal. New York: Oxford University Press.

van Honk, J., Schutter, D. J. L. G., Hermans, E. J., Putman, P., Tuiten, A., \& Koppeschaar, H. (2004). Testosterone shifts the balance between sensitivity for punishment and reward in healthy young women. Psychoneuroendocrinology, 29, 937-943.

van Peer, J. M., Roelofs, K., \& Spinhoven, P. (2008). Cortisol administration enhances the coupling of midfrontal delta and beta oscillations. International Journal of Psychophysiology, 67, 144-150.

van Wingen, G., Mattern, C., Verkes, R. J., Buitelaar, J., \& Fernández, G. (2010). Testosterone reduces amygdala-orbitofrontal cortex coupling. Psychoneuroendocrinology, 35, 105-113.
Varela, F., Lachaux, J. P., Rodriguez, E., \& Martinerie, J. (2001). The brainweb: Phase synchronization and large-scale integration. Nature Reviews Neuroscience, 2, 229-239.

Velikova, S., Locatelli, M., Chiara Insacco, C., Smeraldi, E., Comi, G., \& Leocani, L. (2010). Dysfunctional brain circuitry in obsessive-compulsive disorder: Source and coherence analysis of EEG rhythms. Neuroimage, 49, 977-983.

Viau, V. (2002). Functional cross-talk between the hypothalamicpituitary-gonadal and -adrenal axes. Journal of Neuroendocrinology, 14, 506-513.

Volkow, N. D., Gomez-Mont, F., \& Inamdar, S. (1987). Multivariate analyses of the EEG in normal adolescents. Biological Psychiatry, 22, 199-204.

Wacker, J., Dillon, D. G., \& Pizzagalli, D. A. (2009). The role of the nucleus accumbens and rostral anterior cingulate cortex in anhedonia: Integration of resting EEG, fMRI, and volumetric techniques. Neuroimage, 46, 327-337.

Wood, R. I. (1996). Functions of the steroid-responsive neural network in the control of male hamster sexual behavior. Trends in Endocrinology and Metabolism, 7, 338-344. 